\title{
A Simulation Framework to Assess Pattern Matching Algorithms in a Space Mission
}

\author{
Alessandro Gherardi ${ }^{1}$ and Alessandro Bevilacqua ${ }^{1,2}$ \\ 1 ARCES - Advanced Research Center on Electronic Systems, \\ University of Bologna, Italy \\ agherardi@arces . unibo.it \\ 2 DEIS - Department of Electronics, Computer Science and Systems, \\ University of Bologna, Italy \\ alessandro.bevilacqua@unibo.it \\ http://cvg.deis.unibo.it
}

\begin{abstract}
Within the framework of the European Space Agency (ESA), the BepiColombo space mission will target Mercury as the planet to be studied to discover more about the formation and the composition of the inner planets of our solar system. Mercury exhibits an effect (the libration) whose amplitude could determine whether the core is liquid.

The method we propose to estimate the libration amplitude is based on matching of surface features extracted from image pairs taken at different epochs and thereby affected by illumination and scale artifacts. Since no detailed image pairs are available to assess the accuracy of the methods we propose, in order to generate synthetic images of the planet's surface we have developed the simulation framework we present in this work. Finally, we discuss some preliminary matching results on surface features extracted from our synthetic images taken at different altitude and affected by illumination changes.
\end{abstract}

Keywords: synthetic image, satellite imaging, pattern matching, simulation framework.

\section{Introduction}

The BepiColombo mission is the cornerstone mission of ESA that will target Mercury to discover the formation and the composition of the inner planets of our solar system. The main challenges arise from the planet being very close to the Sun, which affects many technological aspects. Firstly, Mercury is hard to observe from a distance because the Sun is very bright. Furthermore, it is difficult to reach because a spacecraft must loose a lot of energy to approach the planet and enter in its nominal orbit. Moreover, the high gravity field of the Sun presents a challenge in placing the spacecraft into a stable orbit around Mercury. Once in a stable orbit, the spacecraft must also cope with high thermal issue, since its surface temperatures may reach $450^{\circ} \mathrm{C}$. The spacecraft, will be launched in 2014 and will have a interplanetary cruise to Mercury using solarelectric propulsion and by exploiting Moon, Venus and Mercury gravity assists. 
The Mercury Planetary Orbiter (MPO) probe will be captured into polar orbit stabilized in nadir pointing, which will have periherm distance of $400 \mathrm{~km}$ and a apoherm distance of $1500 \mathrm{~km}$, with 2.3 hours orbital period [1. Among the instruments carried by MPO, an imaging system will provide detailed images of the surface of Mercury, through wide angle and narrow angle High Resolution Cameras (HRIC). One of the main objective of the mission is the Mercury Orbiter Radio science Experiment (MORE) [2]. The MORE experiment will help to determine the gravity field of Mercury, as well as the size and physical state of its core. It will provide crucial experimental constraints to the models of the planet's internal structure and test theories of gravity with unprecedented accuracy. The main objective of the rotation experiment is the estimation of Mercury obliquity and libration amplitude: the physical libration of the mantle about the mean resonant angular velocity arises from the periodically reversing torque on the planet as Mercury rotates relative to the Sun. Roughly, such an effect will be seen as an offset in longitude of the same point on the Mercury surface observed by the orbiting probe at different phases of the libration. The amplitude of this libration is approximately equal to $400 \mathrm{~m}$ at the equator, following a sine-like wave as Mercury orbits around the Sun. This knowledge will help geologist and space scientist to determine if Mercury has a molten core [4. Within the framework of the MORE rotation experiment, the estimation of Mercury obliquity and libration amplitude can be accomplished by collecting pictures of Mercury's surface by using the HRIC camera. The Mercury libration and rotation axis can then be measured by analyzing image pairs relative to the same region and taken at different epochs by identifying features in the two images [3]. However, several constraints must be considered for a successful application of the method. For example, the probe will approach Mercury in a polar orbit, which will make the pictures of its surface taken at different altitude, thereby with different scale factors at different resolutions. Moreover, the estimation of the libration amplitude needs to be accomplished at epochs where the amplitude is high enough to be appreciated by the instruments. Here, the difference in phase angle (the angle between the Sun, the point on the surface and the observer) will bring images affected by different illumination conditions. Until now, only NASA Mariner 10 in the mid 70s and now Messenger have been able to acquire close up images of the Mercury surface. The former probe has provided images with a limited resolution of about $45 \%$ coverage of Mercury during the three flybys. The latter will enter into a polar orbit around Mercury soon, giving much more details on the Mercury surface.

In order to study and test the most effective pattern matching algorithms that meet the required accuracy in estimating the libration, a dataset of image pairs sharing the same portion of the planet's surface, taken at different epochs during the orbit, must be considered. The lack of both high resolution and different illumination conditions in the currently available images of the Mercury surface, requires to have at least synthetic images at one's disposal. In this work, we present the simulation environment used to generate synthetically all the possible image pairs. Starting from the generation of an earlier approximation of 
the Mercury surface, more morphological features (mainly craters) are added to the surface to generate the final Digital Elevation Model (DEM). The synthetic image is then raytraced according to the orbital parameters of the imaging system.

\section{Previous Work}

In [5], the authors simulated the pattern matching of albedo features and craters for a Mercury orbiter using synthetically generated images. Their study concluded that sub-pixel accuracy could be achieved, if images would be obtained at phase angles greater than $5 \mathrm{deg}$ and less than $55 \mathrm{deg}$ for albedo spots and if the difference between the phase angles are smaller than $35 \mathrm{deg}$ for craters. The albedo spot features they employed are a resampled version of an albedo map of Deimos, the smaller and outermost of Mars' two natural satellites. Nevertheless, there is no evidence that albedo spots at such a small scale can be found on Mercury. Also, the craters modeled and rendered by authors are used together with a trivial and non automatic pattern matching technique. Also, the restriction imposed by the orbital constraints and Sun phase angles limits the number of image pairs available for the rotation experiment. Authors assume that the overall accuracy required for the libration and rotation estimations can be met, if at least 25 image targets are observed repeatedly over the nominal mission of 360 days.

In [6], the authors simulated the surface of Mercury in order to assess the accuracy of the pattern matching technique. The authors make use of DEMs taken from other planets and a synthetically generated DEM, subsequently raytraced with POV-Ray [8] under different illumination conditions. Results show that a match with sub-pixel accuracy can be attained on $95 \%$ of the trials on real DEMs and only in $87 \%$ of the cases with the synthetic DEM. Also, as authors state, the synthetic DEM suffers from being not sufficiently realistic. The matching of pairs is possible if the Sun elevation (the angle between the Sun position and the surface horizon plane) is not at the zenith, it is greater than $10 \mathrm{deg}$ and the Sun azimuth (the angle between North and the projection of the Sun vector in the horizon reference plane) between the images is less than $30 \mathrm{deg}$.

As far as the surface features are concerned, the authors in [5], 2], 3] have chosen the albedo features as the best candidate for the pattern matching stage. When present, these features are due to changes in the reflectance of the surface material, the opposite of the variations in relief that characterize all the morphological surface features. However, the work in [12] points out that even if small scale albedo spot could be present on the Mercury surface, and even though a global map of albedo spot can be built by examinations of the currently available Mercury images, the size of such features is still prohibitive for the small field of view of the BepiColombo narrow angle camera. 


\section{The Method}

\subsection{The Surface Features Considered}

The albedo features have raised importance due to their photometric appearance being not related to geomorphological structure of the terrain. For a long time these have been desirable features for visual inspection since they allow the observer to identify the place on the planet being watched. Erroneously, their independence from any morphological structure has been considered useful for automatic pointing, or even for automatic tracking. As a matter of fact, the assumption that the albedo features are reliable features that can be employed for accurate pattern matching has to be reconsidered. First, classic albedo features of Mercury are planetary wide, they come from telescope observations and are motivated by visual observation. Accordingly, they are unfeasible to be tracked by a small FOV camera like the one used in the BepiColombo mission, designed to capture surface details. Perhaps, other local, "small scale", albedo features might be present on the Mercury surface although neither their existence nor their location is documented. Second, due to the presence of morphological features like craters and faults, the albedo may undergo shadowing effects as the Sun position changes, thus their appearance changes photometrically, being not invariant for our purposes.

On the other hand, craters are widely spread over the surface of Mercury, similarly to the Moon, although the higher gravitational field of Mercury and its closeness to the Sun implies a higher impact velocity of bodies reaching the surface. The distribution of crater sizes on Mercury extends to a wide range of diameters, from very high impact basins of the order of thousands of kilometers to very small craters of few meters [9]. According to the change in craters' appearance, due to different illumination conditions, a suite of pattern matching algorithms can be built to target each class of differences in illumination. Moreover, as long as these changes keep limited, the pattern matching can be accomplished by using local features tracked between the image pairs, either using the Lukas Kanade feature Tracker (KLT) [14] or more structured feature descriptors such as Scale Invariant Feature Transform (SIFT) [13] which take into account also the difference in scale. Therefore, our primary choice is to target all the surface features present in the images according to each class of changes in illumination and scale. In this work we target craters as the main image features, albeit not directly as pattern matching features.

\subsection{Image Generation}

The simulation system builds a DEM of the Mercury surface by simulating a set of impact craters on an initial terrain model. Craters are randomly distributed on the surface according to a set of parameters defining their diameters and the density distributions. Each crater is modeled by combining impact crater models 11, 90] and surface fractal details.

Mercury presents mainly two types of craters: simple craters, bowl-shaped structures formed by the smallest impact bodies which create sharp rims, and 
complex craters, characterized by terraced walls due to subsurface faults which present a central peak of material brought up from beneath the surface. The transition diameter between simple and complex crater is $10.3 \mathrm{~km}$ according to [11. The craters are modeled by using Gaussian shapes. A number of slightly shifted 2D Gaussian shapes have been superimposed in order to obtain an irregular border on top of the crater (see Fig. 1). These shapes have been cut starting from a $17 \%$ base level added by a random component. The inner side of the crater has been modeled by reversing each Gaussian shape, after doubling its sigma, in order to simulate a smooth impact area. Complex craters have been also modeled by superimposing a central peak in the impact area. Fig. 1] shows

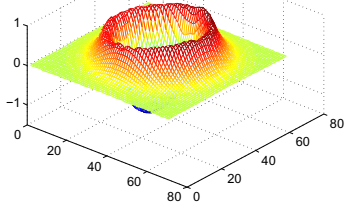

(a)

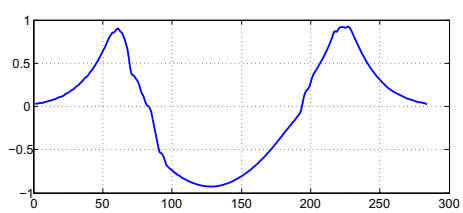

(b)

Fig. 1. Simple crater model (a) and its profile (b)

shape and profile of a simple crater generated by this method. The synthetic image generation procedure is outlined as follows:

1. a fractional Brownian motion (fBm) algorithm generates the plain surface according to a $1 / f^{n}$ pink noise, where $f$ is the frequency and $n$ the argument. This procedure permits to define the coarser texture level free from any other feature (i.e., craters, bumps, scratch, etc...) at each scale;

2. a random number of craters is added to the surface, with variable diameter size according to the parameters given;

3. step 1 and 2 are repeated by a prefixed number of times for each scale, until the resulting DEM is generated.

The first step allows to generate the main coarser irregular surface, and at each iteration it smooths the previous generated DEM in order to simulate different ages of impacts on the surface. This behavior is carried out by increasing the argument of the pink noise, at each scale.

A number of craters is then added randomly to the coarse DEM shape. In particular, the density of craters is within three predefined ranges, according to three different "main diameters" parameters. The latter have been estimated according to 910] and by analyzing the size of craters of images acquired by Messenger and Mariner10 space missions, also taking into account the ground resolution of the BepiColombo HRIC camera. Each "main diameter" pertaining to each generation stage has its own variation parameter which adds a random 
crater diameter within each range. It is worth noticing that, at the time being, no data is available in order to estimate what kind of features would be visible by the HRIC camera sensor, especially at the periherm side. In fact, considering that for a HRIC image the ground size is between about $10 \mathrm{~km}$ and $40 \mathrm{~km}$ at full resolution, no images at such resolution have been acquired by space probes so far. Nevertheless, small impact craters and terrain features, which can be properly modeled by fBm, are likely to be expected. The proposed simulator is able to achieve a balance in the output scene when considering these two components.

The synthetic DEM of Mercury generated by our algorithm is raytraced by a public domain rendering program [8]. At present, the camera is modeled as a pinhole camera, since optical distortions and aberrations will be already corrected in the input images. Generated images are further convolved with a nominal Point Spread Function (PSF) which has been assumed to be Gaussian with a FWHM of 1.1 pixels. This will be effectively modeled after the real camera PSF estimation. The Field Of View (FOV) of the camera is $25 \mathrm{mrad}$, which gives projections of the planet's surface having a ground size between 10 to $40 \mathrm{~km}$ according to the altitude of the probe. The sensor size is of $2048 \times 2048$ pixels at full resolution. The satellite position is expressed in latitude and longitude of the nadir point $O$ (the point on the surface along the vertical direction according to the gravity field), together with the altitude relative to the surface ground. The Sun illumination direction is modeled by two angles: elevation and azimuth.

\section{Results}

\subsection{Synthetic Image Generation}

In Fig. 2, a rendered DEM is presented showing the generation of images with different illumination angles as well as changes in scale (last row). The first row show very a high change in both elevation angle (67 and $20 \mathrm{deg}$ ) and azimuth (141 and $72 \mathrm{deg}$ ). The shadows apper more emphasized on the second image. On the second row, Sun elevation varies slowly (ten degrees) while azimuth angle changes prominently from $12 \mathrm{deg}$ (c) to $224 \mathrm{deg}$ (d). Here we can see how the appearance of craters have been slightly modified. In fact, the projected shadows on the surface change the shape of the crater rims. In the third row, two images of the same DEM have been acquired with varying altitude, (e) being taken at $700 \mathrm{~km}$ and (f) at the nominal distance of the periherm side $(400 \mathrm{~km})$ with a difference of $100 \mathrm{deg}$ in azimuth angles and by keeping constant the elevation angles. From these images it is readily evident how the azimuth angle plays a relevant role in affecting the subsequent pattern matching stage. Craters' shadows are altered both in the inner and outer regions. This behavior, while being almost impractical to be tackled by correlation-based and feature descriptors matching methods, could be faced by shape from shading and combined matching algorithms.

In Fig. 3, the terrain generated is heavily cratered. Also the illumination angle changes slightly from frame to frame. In fact, elevation is near horizon for 

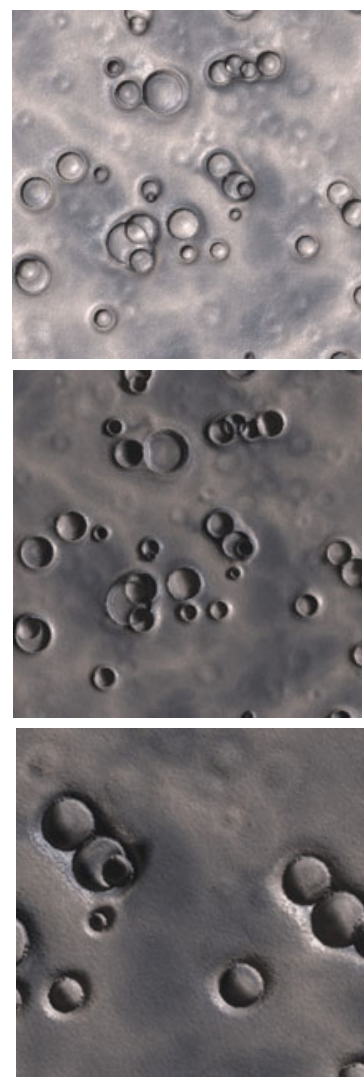

(a)
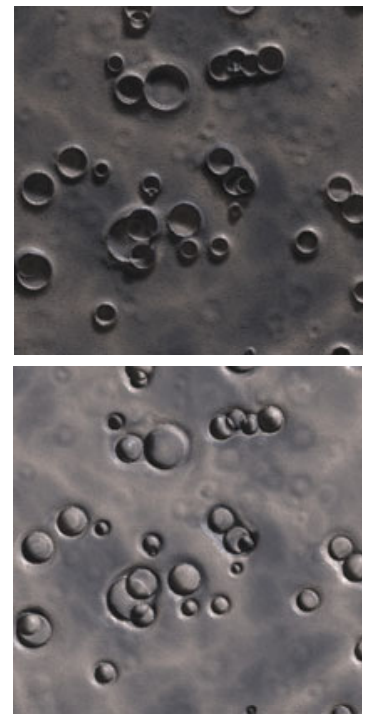

(c)

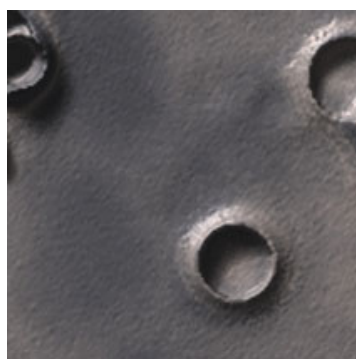

(b)

(d)

(f)

Fig. 2. Simulated images of Mercury surface at different illumination angles
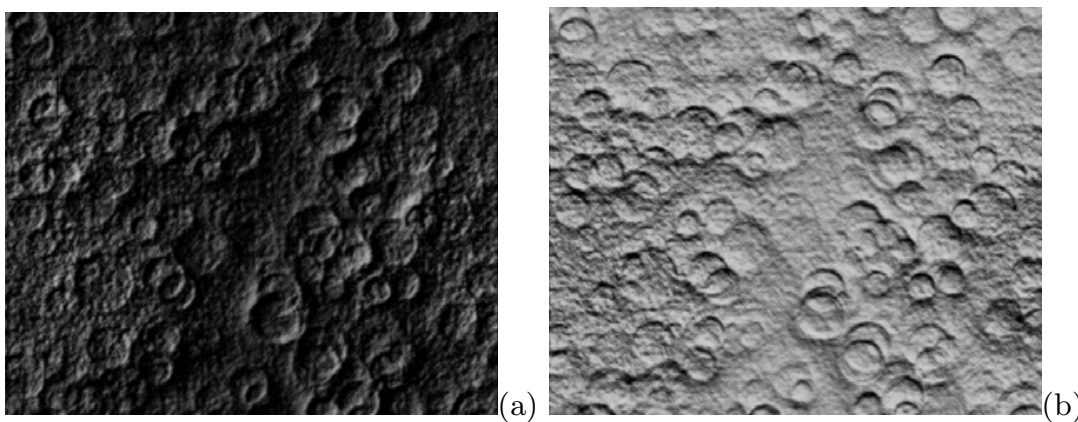

Fig. 3. Simulated images of Mercury surface in a heavily cratered area

the first image and about 42 deg for the second one, whilst azimuth angles are almost in opposition. Here, the shadows projected by craters and surface's erosion make practically every pixel of the two frames unrelated. Thus, also in this example, correlation-based as well as shape-based pattern matching algorithms would likely fail. 


\subsection{Feature Tracking}

Here, a preliminary study on the extraction and matching of features is reported. In Fig. 4(a), the SIFT features extracted and matched from Figs. 2(d) and 2(e) are shown. The two frames show a change in altitude of about $800 \mathrm{~km}$, a change in illumination angles of $10 \mathrm{deg}$ for elevation and no change in illumination

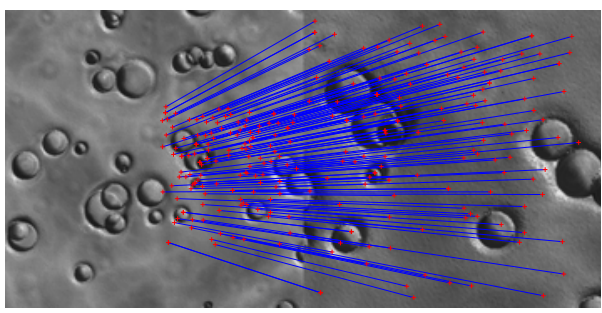

(a)

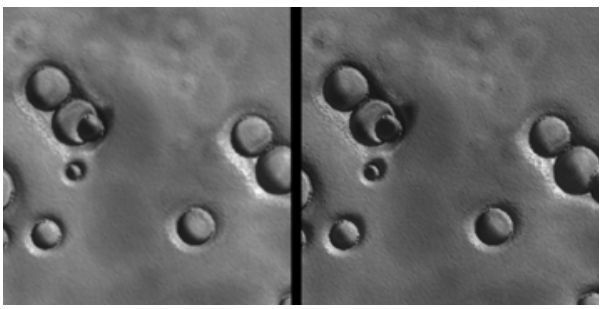

(b)

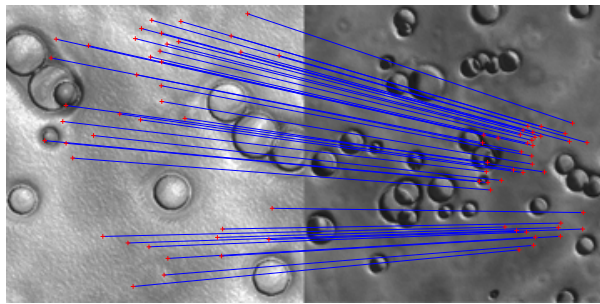

(c)

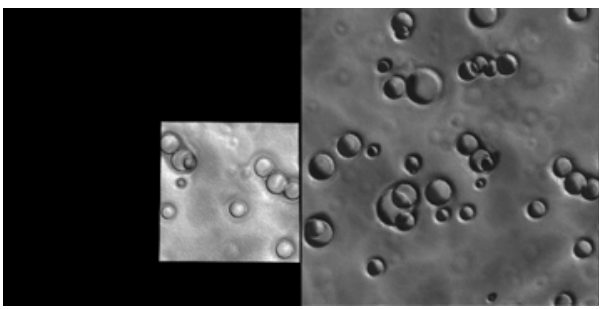

(d)

Fig. 4. Matched SIFT features between two frame couples (a,c) taken at different scale and illumination angles: difference in elevation is $10 \mathrm{deg}$ in (a) and more than $60 \mathrm{deg}$ in (c). In (b,d) the first frame is reprojected according to the estimated $H$ (see text). 
azimuth. Since the camera moves according to the known orbital parameters and from that altitude an approximation of a planar surface with respect to the small FOV could be made, the two views are related by an homography transformation, which maps points in the first frame to the corresponding points on the second frame. In a more realistic case, also the dispalcement of the camera centers will be included into the transformation model, thereby relaxing the panar surface assumption. The homography $H$ recovered by using RANSAC 15 is used to estimate the scale and the position of the corresponding pixel in the two images. The first image reprojected on the domain of the second is shown in Fig. 4(b). The estimation accuracy is quite good, being the imposed offset (133 and 59 pixel in the horizontal and vertical directions, respectively) effectively recovered with sub-pixel accuracy, even when elevation angles vary of more than 60 deg (Fig. 4 (c,d)). However, as expected, trials conducted with very low or very high elevations and with changes in the azimuth angle show that this feature matching fails. These are among the numerous cases that have to be tackled with an ensemble of matching algorithms built on purpose.

\section{Conclusion}

The BepiColombo rotation experiment, which aims at estimating the obliquity and libration of Mercury, is a crucial experiment in order to assess many important implications on our solar system. The recovery of the libration amplitude can be achieved by matching surface features extracted from image pairs taken at different epochs and thereby affected by illumination and scale artifacts. The lack of detailed image pairs available from other space missions to Mercury limits the possibility to assess the accuracy of the methods we propose. In this work a simulation framework aiming to both generate realistic images of the Mercury surface as well as devising pattern matching algorithms is presented. The framework is able to generate synthetic images according to the orbital parameters of the space mission, thereby allowing to test the pattern matching algorithms under different scale and illumination conditions, as close as possible to the real case scenario. Finally, some preliminary matching results dealing with surface features extracted from our synthetic images taken at different altitude and affected by illumination changes show that sub-pixel accuracy is possible even for high changes in Sun elevation angle. Also, as expected, trials conducted with very low or very high elevations and with changes in the azimuth angle have to be tackled with an ensemble of matching algorithms built on purpose.

\section{References}

1. Schulz, R., Benkhoff, J.: BepiColombo: Payload and mission updates. Advances in Space Research 38(4), 572-577 (2006)

2. Iess, L., Boscagli, G.: Advanced radio science instrumentation for the mission BepiColombo to Mercury. Planet. Space Sci. 49, 1567-1608 (2001) 
3. Iess, L., Asmar, S., Tortora, P.: MORE: An advanced tracking experiment for the exploration of Mercury with the mission BepiColombo. Acta Astronautica 65, 666-675 (2009)

4. Jehn, R., Corral, C., Giampieri, G.: Estimating Mercury's 88-day libration amplitude from orbit. Planetary and Space Science 52(8), 727-732 (2004)

5. Jorda, L., Thomas, N.: The accuracy of pattern matching techniques for the radio science experiment of ESA's Mercury Cornerstone mission. Preliminary study at Max-Planck Institute for Aeronomie, Katlenburg-Lindau, Germany (2000) (unpublished)

6. Vacanti, G., Buis, E.J., Beijersbergen, M.: BepiColombo: Study on Techniques and Accuracies of Pattern Matching of Remote Sensing Images. Tech. rep., Cosine Research ESA (2005)

7. Peale, S.J., Yseboodt, M., Margot, J.: Long-period forcing of Mercurys libration in longitude. Icarus 187, 365-373 (2007)

8. Persistence of Vision Raytracer (2004), http://www.povray.org/

9. Gault, D.E., Guest, J.E., Murray, J.B., Dzurisin, D., Malin, M.C.: Some Comparisons of Impact Craters on Mercury and the Moon. J. Geophys. Res. 80(17), 2444-2460 (1975)

10. Melosh, H.J., Ivanov, B.A.: Impact Crater Collapse. Earth Planet. Sci. 27, 385-415 (1999)

11. Pike, R.J.: In: Vilas, F., Chapman, C.R., Matthews, M.S. (eds.) Mercury, pp. 165273. University of Arizona Press, Tucson (1988)

12. Denevi, B.W., Robinson, M.S.: Mercury's albedo from Mariner 10: Implications for the presence of ferrous iron. Icarus 197(1), 239-246 (2008)

13. Lowe, D.G.: Distinctive image features from scale-invariant keypoints. International Journal of Computer Vision 60, 91-110 (2004)

14. Tomasi, C., Kanade, T.: Detection and Tracking of Point Features. Carnegie Mellon University Technical Report CMU-CS-91-132 (1991)

15. Fischler, M.A., Bolles, R.C.: Random sample and consensus: A paradigm for model fitting with application to image analysis and automated cartography. Comm. of the ACM 24, 381-395 (1981) 